\section{OPEN ACCESS}

Edited by:

Nicola Montano,

University of Milan, Italy

Reviewed by: Valdo Jose Dias Da Silva, Universidade Federal do Triângulo

Mineiro, Brazil

Eleonora Tobaldini,

University of Milan, Italy

${ }^{*}$ Correspondence:

Lilei Yu

lileiyu@whu.edu.cn

Hong Jiang

hong-jiang@whu.edu.cn

†These authors have contributed equally to this work

Specialty section: This article was submitted to General Cardiovascular Medicine, a section of the journal Frontiers in Cardiovascular Medicine

Received: 27 April 2021 Accepted: 02 July 2021 Published: 27 July 2021

Citation:

Wang J, Liu W, Chen H, Liu C, Wang M, Chen H, Zhou H, Liu Z,

Zhang S, Yu Z, Duan S, Deng Q, Sun J, Jiang $H$ and $Y u L$ (2021) Novel Insights Into the Interaction Between the Autonomic Nervous System and Inflammation on Coronary Physiology: A Quantitative Flow Ratio Study. Front. Cardiovasc. Med. 8:700943. doi: 10.3389/fcvm.2021.700943

\title{
Novel Insights Into the Interaction Between the Autonomic Nervous System and Inflammation on Coronary Physiology: A Quantitative Flow Ratio Study
}

\begin{abstract}
Jun Wang ${ }^{\dagger}$, Wei Liu ${ }^{\dagger}$, Huaqiang Chen, Chengzhe Liu, Meng Wang, Hu Chen, Huixin Zhou, Zhihao Liu, Song Zhang, Zhongyang Yu, Shoupeng Duan, Qiang Deng, Ji Sun, Hong Jiang* and Lilei Yu*

Department of Cardiology, Renmin Hospital of Wuhan University, Cardiac Autonomic Nervous System Research Centre of Wuhan University, Cardiovascular Research Institute, Wuhan University, Hubei Key Laboratory of Cardiology, Wuhan, China
\end{abstract}

Background: Heart rate variability (HRV) was proposed as a noninvasive biomarker to stratify the risk of cardiovascular disease. However, it remains to be determined if HRV can be used as a surrogate for coronary artery physiology as analyzed by quantitative flow ratio (QFR) in patients with new-onset unstable angina pectoris (UAP).

Methods: A total of 129 consecutive patients with new-onset UAP who underwent 24-h long-range 12-channel electrocardiography from June 2020 to December 2020 were included in this study. HRV, coronary angiography, and QFR information was retrieved from patient medical records, the severity of coronary lesions was evaluated using the Gensini score (GS), and total atherosclerotic burden was assessed using the three-vessel contrast QFR (3V-cQFR) calculated as the sum of cQFR in three vessels.

Results: Multivariate logistic analysis showed that low-frequency power (LF) and high-sensitivity C-reactive protein (hs-CRP) were directly correlated with functional ischemia of target vessel, which were inversely correlated with total atherosclerotic burden as assessed by 3V-cQFR. Moreover, incorporation of the increase in LF into the existing model that uses clinical risk factors, GS, and hs-CRP significantly increased the discriminatory ability for evaluating coronary artery physiology of target vessel.

Conclusions: LF and hs-CRP are independently associated with functional ischemia in patients with new-onset UAP. The relative increase of LF and hs-CRP could add value to the use of classical cardiovascular risk factors to predict the functional severity of coronary artery stenosis. Our results suggest a potential association between the autonomic nervous system, inflammation, and coronary artery physiology.

Keywords: unstable angina pectoris, quantitative flow ratio, heart rate variability, autonomic nerve, inflammation 


\section{INTRODUCTION}

Traditional cardiovascular risk factors, such as age, family history of premature heart disease, diabetes, cigarette smoking, hypertension, and dyslipidemia, have been demonstrated to be linked to the incidence of acute cardiovascular events and the increased risks of morbidity, mortality, and disability in patients with acute coronary syndrome (ACS) (1). However, acute coronary events can occur in healthy individuals without the above-mentioned traditional cardiovascular risk factors, suggesting the presence of unrecognized risk factors $(1,2)$. Pathologically, the incidence of acute coronary events has been associated with functionally significant coronary artery stenosis and total physiological atherosclerotic burden (3-6). Therefore, identifying hidden risk factors correlated with functional severity of coronary artery stenosis and total atherosclerotic burden would be important for predicting acute coronary events in patients with ACS, particularly in those with unstable angina pectoris (UAP).

Strikingly, the heart rate variability (HRV) measurement, a simple and non-invasive technique that provides assessment of autonomic modulation of cardiac regulation, can independently predict the mortality of patients with no known history of cardiovascular disease (7). HRV has been documented in patients at higher risk for ACS, such as those with diabetes, hypertension, and high low-density lipoprotein cholesterol (8-11). Moreover, low-frequency power (LF) is highly predictive for coronary artery disease (CAD) regardless of the traditional cardiovascular risk factors, and it is a potential clinical risk stratification tool in patients with sinus rhythm (12). Clinical studies have also demonstrated an inverse correlation between HRV and chronic low-grade systemic inflammation in patients with stable CAD (13). Accumulating evidence demonstrates that the autonomous nervous system (ANS) plays an important role in the regulation of systemic inflammation, and an ANS imbalance may contribute to the increased risk of acute cardiovascular events via promoting inflammation and endothelium damage (14-16). However, whether the balance of the ANS evaluated by HRV and inflammation is independently associated with functionally significant coronary artery stenosis in patients with ACS remains to be determined.

Compared to the traditional coronary angiogram, quantitative flow ratio (QFR) has become a popular tool to more accurately evaluate the functional severity of coronary artery stenosis based on three-dimensional quantitative angiography and fluid dynamics algorithms (17). The QFR measurement does not require the use of pressure-wires, hyperemia induction, or reconstruction of all side branches, compared to fractional flow reserve (FFR) (18). Moreover, in terms of diagnosing functional coronary artery stenosis, QFR is highly consistent with FFR, the gold standard for evaluating functional stenosis of coronary arteries (18). Although the association between HRV, inflammation, and the severity of coronary angiographic stenosis has been confirmed $(12,19)$, there remains no literature providing any evidence that HRV and inflammation are correlated with the severity of functional coronary artery stenosis as detected by QFR. The aim of the current study was to systematically evaluate the potential association between HRV, inflammation, and coronary physiology in new-onset UAP patients.

\section{METHODS}

\section{Patient Population}

A total of 129 consecutive patients with a first diagnosis of new-onset UAP and no known history of CAD who underwent coronary angiography and QFR measurement at the Department of Cardiology of Renmin Hospital of Wuhan University from June 2020 to December 2020 were included in this retrospective study. Diagnosis of UAP was made in accordance with previously established guidelines (20). Patients were excluded if they had the following conditions: previous paroxysmal or atrial fibrillation, implantation of pacemaker, other arrhythmias (e.g., frequent premature beat, bradycardia, sick sinus syndrome, atrioventricular block, broad bundle branch blocks, or ventricular arrhythmias), depressive disorder, hyperthyroidism, excessive drinking, inflammatory disease, malignant tumors, acute or chronic infection, any systemic acute disease, taking any medications affecting HRV, history of CAD, variable angina, aggravated effort type angina pectoris, valvular heart disease, and congenital heart disease. For QFR analysis, patients with either a prolonged occluded coronary bypass graft, myocardial bridge, ostial lesions, severe vessel overlap or tortuosity at the stenotic segments, side branch lesions, or poor angiographic image quality where QFR measurement could not be constructed were excluded. Because this was a retrospective observational study, the Renmin Hospital of Wuhan University Ethics Committee granted an exemption from requiring ethics approval and informed consent from eligible patients was waived.

\section{Blood Tests}

Venous blood samples were obtained upon admission from each patient before coronary angiography and analyzed for lipid profile, inflammation biomarkers (high-sensitivity C-reactive protein (hs-CRP), interleukin 2 (IL-2), IL-4, IL-6, IL-10, and fibrinogen), and other blood biochemical routine tests at the Department of Clinical Laboratory in Renmin Hospital of Wuhan University.

\section{Holter Monitoring and HRV Analysis}

For all participants, a 24-h long-range 12-channel electrocardiography recording was applied for HRV analysis before coronary angiography. The $24-\mathrm{h}$ mean heart rate and variables of HRV were automatically analyzed using commercially available software (H-Scribe Analysis System, Mortara Instrument, Inc., Milwaukee, WI, USA). The specific signal processing steps for computation of the HRV parameters were the same as described previously (21-23). The frequency domain analysis method was used to analyze the 5-minute shortterm with relatively stable RR interval by Fourier transform, where the power spectrum graph was created using frequency $(\mathrm{Hz})$ as the abscissa coordinate and power spectral density as the ordinate coordinate. The average 5-minute short-term HRV analysis was performed in our study. The spectrum of 
the short-range record is divided into three frequency bands, where VLF, LF, and HF are the integrated power spectral density (PSD) values in different frequency bands. (1) TP (total power): total frequency, frequency spectra $\leq 0.4 \mathrm{~Hz}$, representing total variations of normal-to-normal R-R intervals
(NN intervals), (2) HF (high frequency power): high frequency, frequency spectra $0.15-0.4 \mathrm{~Hz}$, (3) LF (low frequency power): low frequency, frequency spectra $0.04-0.15 \mathrm{~Hz}$, (4) very low-frequency (VLF): very low frequency, frequency spectra $0.003-0.04 \mathrm{~Hz}$. Absolute spectral power values are expressed in

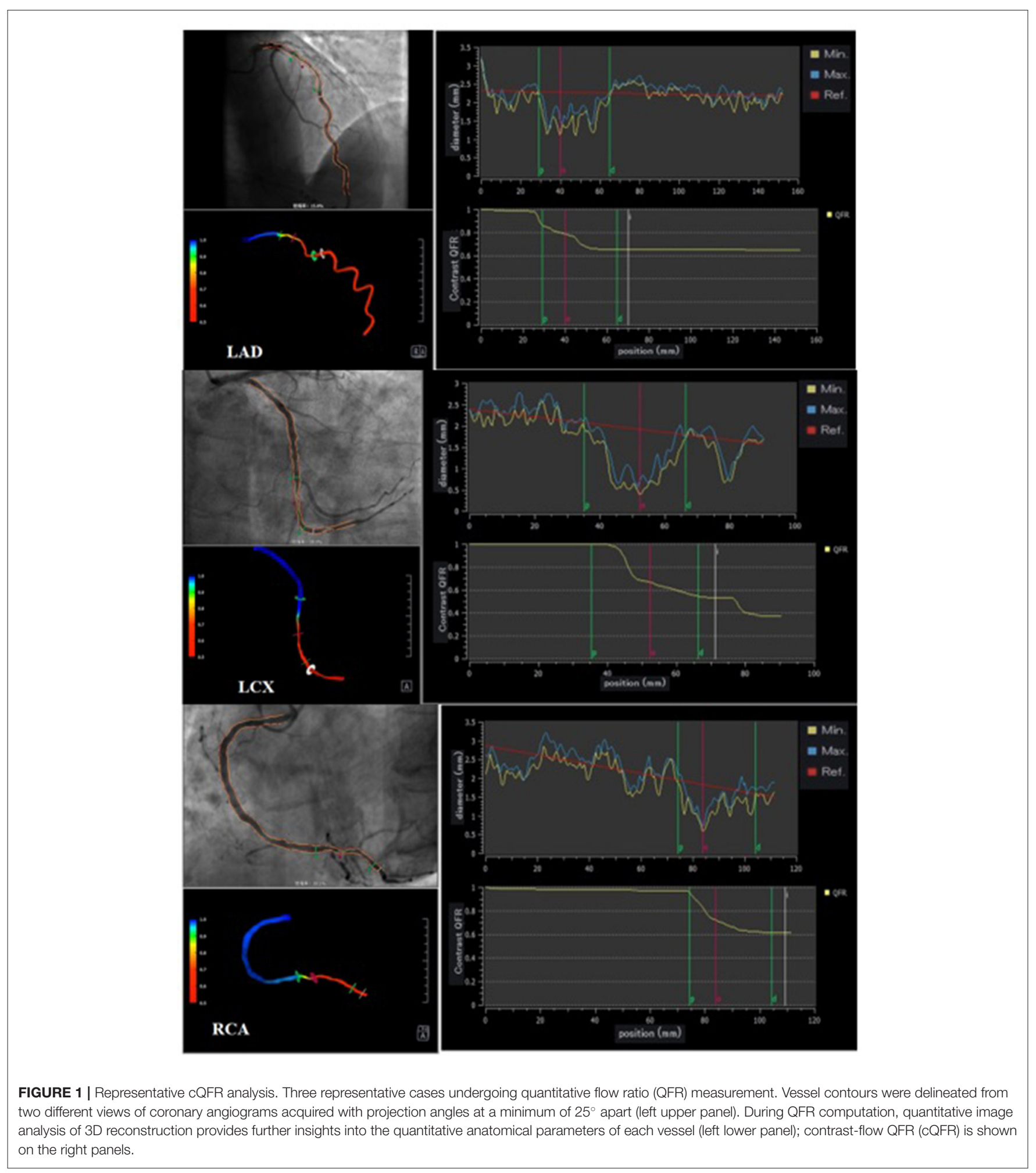


squared milliseconds $\left(\mathrm{ms}^{2}\right)$. Normalized LF power was calculated as LFn $=100^{*} \mathrm{LF} /($ total power-VLF), and normalized HF power was calculated as $\mathrm{HFn}=100^{*} \mathrm{HF} /($ total power-VLF). HF and HFn generally reveal cardiac parasympathetic nerve modulation, while LF and LFn are possibly correlated to sympathetic modulation or to autonomic balance. Accordingly, the LF/HF ratio is an indicator of the balance of the cardiac autonomic nervous system (24).

\section{Coronary Angiography and Gensini Score (GS)}

All participants were scheduled to undergo coronary artery angiography. The severity of stenosis of the coronary artery was independently evaluated by two investigators, and consensus was required with a third investigator if any disagreements arose. Target vessel was defined as the vessel with the most severe lesion. The GS, which integrates the extent of luminal narrowing and the geographic importance of the lesion, was calculated to reflect the severity of coronary artery lesions (25).

\section{QFR Computation}

Three vessels in each patient were analyzed using offline QFR and computed using the AngioPlus system (Pulse Medical Imaging Technology, Shanghai, China). The two selected views with a projection angle of a minimum of $25^{\circ}$ apart in the angiographic image were transferred to the QFR system through

TABLE 1 | Patient characteristics according to CQFR of target vessel.

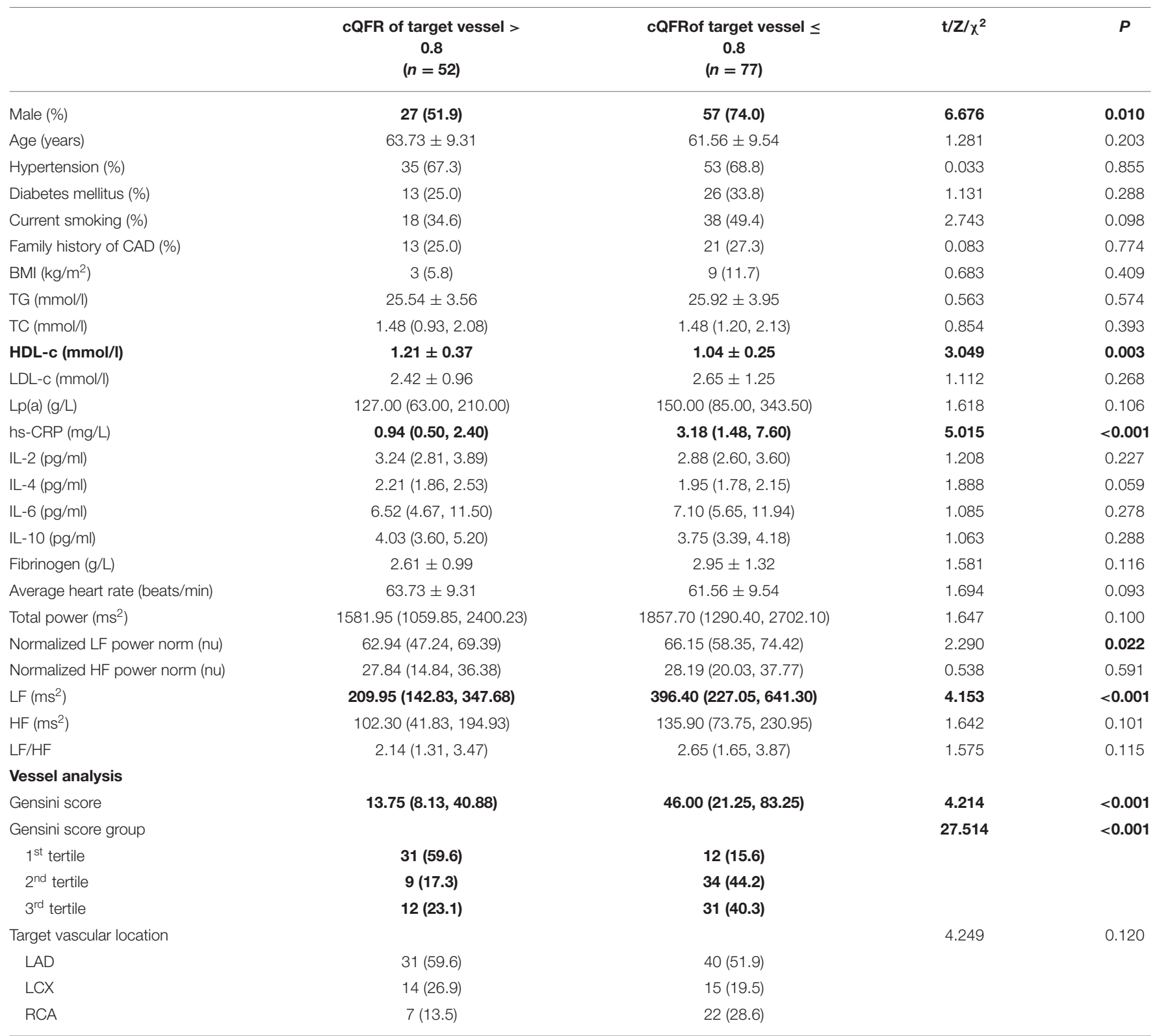

The meaning of the bold values are a two-sided $P<0.05$ was considered statistically significant. 
the local network. A modified TIMI frame count method was used for QFR computation. Recent evidence indicates that in the absence of drug-induced hyperemia, the cQRF obtained through modeled hyperemic flow velocity of the coronary artery shows good consistency with the gold standard FFR $(17,26)$. In our study, cQFR that was obtained from routine angiographic images was used to assess the severity of the functional coronary lesion. Three-vessel cQFR (3V-cQFR) calculated as the sum of cQFR in three vessels has been shown to reflect the total atherosclerotic burden, a variable that can be used to identify "vulnerable" patients (6). Representative examples of the quantitative image analysis of three-dimensional reconstruction and QFR computation are demonstrated in Figure $\mathbf{1 .}$

\section{Statistical Analysis}

Patients were grouped according to the tertiles of the GS. All statistical analyses were performed using SPSS23 software. Comparisons between two groups were performed using an independent sample $t$-test or the Mann-Whitney $U$ test. Categorical data are presented as counts (proportions) and were compared using the $\chi^{2}$ test or Fisher's exact test. Multiple stepwise logistics regression analysis was performed to identify factors associated with the GS and target vessel with QFR $\leq 0.8$. Linear regression analysis was used to identify factors associated with the $3 \mathrm{~V}$-cQFR. ROC curve analysis was used to show the predictability of target vessel with QFR $\leq 0.8$ using GS, hs-CRP, and LF. We compared whether adding GS, hs-CRP, and LF to the traditional cardiovascular risk factors would improve the discriminant and reclassification ability of the models. Continuous data are presented as mean \pm standard deviation (SD) or median (interquartile range), and categorical data are presented as numbers and percentages. A two-sided $P<$ 0.05 was considered statistically significant.

\section{RESULTS}

\section{Patient Characteristics According to cQFR of Target Vessel}

The baseline features of patients based on CQFR of target vessel (cQFR of target vessel $\leq 0.8, n=77$ and $\mathrm{cQFR}$ of target vessel $>0.8, n=52$ ) are presented in Table 1 . Those with cQFR $\leq$ 0.8 of target vessel were mostly male, with lower high-density lipoprotein cholesterol (HDL-C), and higher hs-CRP, GS, LFn, and LF compared to those with $\mathrm{CQFR}>0.8$ of target vessel. There were no correlations between other indicators and cQFR of target vessel $\leq 0.8$ (all $P>0.05$ ). LF was positively correlated with hs-CRP ( $r=0.272, P<0.01$; Figure 2$)$. Multivariable stepwise logistic regression analysis showed that both hs-CRP and LF were independent predictors of functional severity of coronary stenosis of target vessel (all $P<0.05$; Table 2).

\section{Patient Characteristics According to GS}

The baseline features of patients based on the tertiles of GS were $1^{\text {st }}$ tertile GS $<16.5, n=43 ; 2^{\text {nd }}$ tertile GS: $16.5-52.5, n=43$; and $3^{\text {rd }}$ tertile GS $>52.5, n=43$ (Table 3 ). The percentages of being male, current smoking, HDL-C, hs-CRP, IL-6, 3V-cQFR, and CQFR of target vessel were significantly different among the

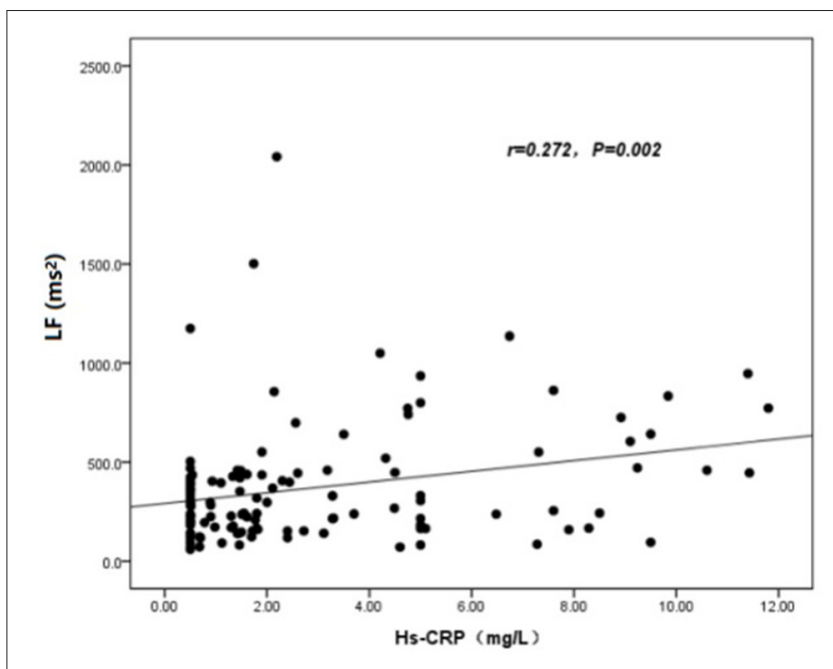

FIGURE 2 | Association analysis between hs-CRP and LF.

groups based on GS tertiles (all $P<0.05$ ). However, there was no correlation between other indicators and the severity of coronary lesions (all $P>0.05$ ). Multivariable stepwise logistic regression analysis showed that both HDL-C and hs-CRP were independent predictors of GS (all $P<0.05$; Table 4).

\section{Linear Regression Model for 3V-cQFR}

The baseline features of patients based on $3 \mathrm{~V}$-cQFR are shown in Table 5. Those with lower 3V-cQFR were mostly male, current smoker, with a family history of CAD, lower HDL-C, and higher hs-CRP, fibrinogen, and LF (all $P<0.05$; Table 5). Multivariate linear regression analysis showed that a family history of CAD, hs-CRP, and LF were all independently and inversely associated with 3 V-cQFR (all $P<0.05$; Table 5). LF $(r=0.444, P<0.001$; Figure 3$)$ and hs-CRP $(r=0.562, P<0.001$; Figure 4).

\section{Evaluation of Discrimination and Reclassification Abilities of the Predictive Models for the Functional Severity of Coronary Stenosis of Target Vessel Using CQFR}

ROC curve analysis showed that GS was predictive of the functional severity of coronary stenosis of target vessel as detected by cQFR, and a cut-off value of GS $=17.00$ conferred a sensitivity of $84.4 \%$ and a specificity of $63.5 \%$. The area under the ROC (AUC) was 0.719 for GS, suggesting good validity $(P<$ $0.001 ; 95 \%$ confidence interval (CI): 0.623-0.815). Hs-CRP was predictive of the functional severity of coronary artery stenosis of target vessel as detected by CQFR, and a cut-off value of hs$\mathrm{CRP}=1.45$ conferred a sensitivity of $79.2 \%$ and a specificity of $59.5 \%$. The AUC for hs-CRP was 0.759 , indicating good validity $(P<0.001 ; 95 \%$ CI: $0.676-0.843)$. LF was predictive of the functional severity of coronary artery stenosis of target vessel as detected by cQFR, and a cut-off value of LF $=390.05$ conferred a sensitivity of $51.9 \%$ and a specificity of $82.7 \%$. The 
TABLE 2 | Independent predictors of cQFR of target vessel $\leq 0.8$.

\begin{tabular}{|c|c|c|c|c|c|c|c|}
\hline \multirow[t]{2}{*}{ Independent variables } & \multirow[t]{2}{*}{ B } & \multirow[t]{2}{*}{ SE } & \multirow[t]{2}{*}{ WALS } & \multirow[t]{2}{*}{$P$} & \multirow[t]{2}{*}{ OR } & \multicolumn{2}{|c|}{$95 \%$} \\
\hline & & & & & & Lower limit & Upper limit \\
\hline Female & 0.297 & 0.519 & 0.328 & 0.567 & 1.346 & 0.487 & 3.722 \\
\hline HDL-C & -1.581 & 0.869 & 3.308 & 0.069 & 0.206 & 0.037 & 1.131 \\
\hline hs-CRP & 0.196 & 0.086 & 5.141 & 0.023 & 1.216 & 1.027 & 1.441 \\
\hline LF & 0.004 & 0.001 & 8.043 & 0.005 & 1.004 & 1.001 & 1.006 \\
\hline
\end{tabular}

The meaning of the bold values are a two-sided $P<0.05$ was considered statistically significant.

TABLE 3 | Patient characteristics according to Gensini Score.

\begin{tabular}{|c|c|c|c|c|c|}
\hline & $\begin{array}{c}1^{\text {st }} \text { tertile }<16.5 \\
(n=43)\end{array}$ & $\begin{array}{l}2^{\text {nd }} \text { tertile } 16.5-52.5 \\
\qquad(n=43)\end{array}$ & $\begin{array}{c}3^{\text {rd }} \text { tertile }>52.5 \\
(n=43)\end{array}$ & $F / Z / \chi^{2}$ & $P$ \\
\hline Male (\%) & $22(51.2)$ & $29(67.4)$ & $33(76.7)$ & 6.348 & 0.042 \\
\hline Age (years) & $60.09 \pm 8.64$ & $64.74 \pm 9.09$ & $62.47 \pm 10.25$ & 2.660 & 0.074 \\
\hline Hypertension (\%) & $33(76.7)$ & $30(69.8)$ & $25(58.1)$ & 3.504 & 0.173 \\
\hline Diabetes mellitus (\%) & $11(25.6)$ & $15(34.9)$ & $13(30.2)$ & 0.882 & 0.643 \\
\hline Current smoking (\%) & $12(27.9)$ & $20(46.5)$ & $24(55.8)$ & 7.068 & 0.029 \\
\hline Family history of CAD (\%) & $5(11.6)$ & $3(7.0)$ & $4(9.3)$ & 0.474 & 0.624 \\
\hline BMI $\left(\mathrm{kg} / \mathrm{m}^{2}\right)$ & $26.19 \pm 3.61$ & $25.71 \pm 3.73$ & $25.39 \pm 4.05$ & 0.345 & 0.710 \\
\hline $\mathrm{TG}(\mathrm{mmol} / \mathrm{l})$ & $1.52(0.93,2.32)$ & $1.42(1.17,1.88)$ & $1.49(1.25,2.11)$ & 0.390 & 0.823 \\
\hline $\mathrm{TC}(\mathrm{mmol} / \mathrm{l})$ & $4.48 \pm 1.35$ & $4.12 \pm 1.23$ & $4.33 \pm 1.39$ & 0.812 & 0.446 \\
\hline HDL-c (mmol/l) & $1.22 \pm 0.40$ & $1.09 \pm 0.26$ & $1.02 \pm 0.22$ & 4.801 & 0.010 \\
\hline LDL-c (mmol/l) & $2.57 \pm 1.18$ & $2.36 \pm 0.94$ & $2.75 \pm 1.27$ & 1.243 & 0.292 \\
\hline Lp(a) $(g / L)$ & $121.50(61.50,287.75)$ & $165.00(89.00,332.00)$ & $139.00(81.00,263.00)$ & 2.175 & 0.337 \\
\hline hs-CRP (mg/L) & $1.47(0.50,3.30)$ & $1.70(0.68,3.28)$ & $4.75(1.10,9.10)$ & 11.012 & 0.004 \\
\hline IL-2 (pg/ml) & $3.11(2.73,3.36)$ & $2.84(2.59,3.42)$ & $3.26(2.82,4.20)$ & 3.543 & 0.170 \\
\hline IL-4 (pg/ml) & $2.21(1.86,2.51)$ & $1.95(1.73,2.28)$ & $1.97(1.78,2.21)$ & 4.277 & 0.118 \\
\hline IL-6 (pg/ml) & $5.19(4.29,8.14)$ & $6.81(5.64,8.83)$ & $11.93(6.70,16.67)$ & 22.672 & $<0.001$ \\
\hline IL-10 (pg/ml) & $3.96(3.60,4.42)$ & $3.68(3.37,4.96)$ & $3.89(3.42,4.85)$ & 0.547 & 0.761 \\
\hline Fibrinogen (g/L) & $2.71 \pm 1.27$ & $2.84 \pm 1.23$ & $2.90 \pm 1.15$ & 0.276 & 0.759 \\
\hline Average heart rate (beats/min) & $68.21 \pm 10.07$ & $67.74 \pm 8.61$ & $69.30 \pm 6.84$ & 0.371 & 0.690 \\
\hline Total power $\left(\mathrm{ms}^{2}\right)$ & $\begin{array}{c}1814.10 \\
(1092.48,2560.40)\end{array}$ & $\begin{array}{c}1934.60 \\
(1202.40,2692.90)\end{array}$ & $\begin{array}{c}1754.00 \\
(1294.80,2590.50)\end{array}$ & 0.151 & 0.927 \\
\hline Normalized LF power norm (nu) & $63.23(48.14,70.57)$ & $67.20(55.02,74.54)$ & $63.33(55.56,73.92)$ & 1.874 & 0.392 \\
\hline Normalized HF power norm (nu) & $26.60(15.18,36.73)$ & $27.46(20.58,35.30)$ & $29.84(19.28,38.24)$ & 1.629 & 0.443 \\
\hline $\mathrm{LF}\left(\mathrm{ms}^{2}\right)$ & $242.20(152.40,422.80)$ & $286.00(165.40,456.20)$ & $359.40(227.50,725.50)$ & 3.816 & 0.167 \\
\hline $\mathrm{HF}\left(\mathrm{ms}^{2}\right)$ & $119.80(51.10,185.10)$ & $95.10(66.70,189.70)$ & $165.00(89.60,256.00)$ & 4.580 & 0.101 \\
\hline LF/HF & $2.37(1.43,3.53)$ & $2.57(1.67,4.07)$ & $2.49(1.57,3.83)$ & 0.741 & 0.690 \\
\hline \multicolumn{6}{|l|}{ Vessel analysis } \\
\hline cQFR of target vessel & $0.78 \pm 0.25$ & $0.67 \pm 0.20$ & $0.62 \pm 0.22$ & 6.183 & 0.003 \\
\hline Three-vessel cQFR & $2.69 \pm 0.34$ & $2.49 \pm 0.35$ & $2.22 \pm 0.44$ & 16.563 & $<0.001$ \\
\hline Target vascular location & & & & 6.539 & 0.162 \\
\hline LAD & $27(62.8)$ & $18(41.9)$ & $26(60.5)$ & & \\
\hline LCX & 7 (16.3) & $11(25.6)$ & $11(25.6)$ & & \\
\hline $\mathrm{RCA}$ & $9(20.9)$ & $14(32.6)$ & $6(14.0)$ & & \\
\hline
\end{tabular}

The meaning of the bold values are a two-sided $P<0.05$ was considered statistically significant.

AUC for LF was 0.716, indicating good validity $(P<0.001$; 95\% CI: 0.628-0.804) (Figure 5). In the multivariable analysis model, GS, hs-CRP, and LF increased the discriminatory indices when added to clinical risk factors (Figure 6). In model 3, the increase of GS also significantly increased the ability to accurately predict the functional severity of coronary artery stenosis of target vessel compared with model 2 using conventional cardiovascular risk factors (AUC: 0.828; C-index: 0.844; Youden index: 0.568 ; sensitivity: $81.8 \%$; specificity: $75.0 \%$; $P<0.001$ ). For the predictability of the functional severity of coronary stenosis of target vessel, the positive Youden index of the combined hsCRP increased in model 4 (AUC: 0.881; C-index: 0.897; Youden index: 0.672 ; sensitivity: $89.6 \%$; specificity: $73.1 \%$; $P<0.001$ ) (Figure 6). Adding LF $>390.05$ into model 5 further increased 
TABLE 4 | Factors associated with the severity of coronary lesions as detected by Gensini Score.

\begin{tabular}{|c|c|c|c|c|c|c|c|}
\hline \multirow[t]{2}{*}{ Independent variables } & \multirow[t]{2}{*}{ B } & \multirow[t]{2}{*}{ SE } & \multirow[t]{2}{*}{ WALS } & \multirow[t]{2}{*}{$\boldsymbol{P}$} & \multirow[t]{2}{*}{ OR } & \multicolumn{2}{|l|}{$95 \%$} \\
\hline & & & & & & Lower limit & Upper limit \\
\hline Female & -0.150 & 0.459 & 0.107 & 0.744 & 0.861 & 0.350 & 2.117 \\
\hline Current smoking & 0.345 & 0.425 & 0.657 & 0.418 & 1.412 & 0.613 & 3.248 \\
\hline HDL-c & -1.685 & 0.681 & 6.132 & 0.013 & 0.185 & 0.049 & 0.704 \\
\hline hs-CRP & 0.065 & 0.033 & 3.974 & 0.046 & 1.067 & 1.001 & 1.138 \\
\hline IL-6 & -0.008 & 0.008 & 1.116 & 0.291 & 0.992 & 0.977 & 1.007 \\
\hline
\end{tabular}

The meaning of the bold values are a two-sided $P<0.05$ was considered statistically significant.

TABLE 5 | Patient characteristics according to baseline 3V-cQFR.

\begin{tabular}{|c|c|c|c|c|c|c|}
\hline & \multicolumn{3}{|c|}{ Univariate } & \multicolumn{3}{|c|}{ Multivariate } \\
\hline & B & $\mathbf{T}$ & $p$ & B & $\mathbf{T}$ & $p$ \\
\hline Female (\%) & 0.286 & 3.851 & $<0.001$ & 0.044 & 0.472 & 0.638 \\
\hline Age (years) & 0.004 & 1.052 & 0.295 & & & \\
\hline Hypertension (\%) & -0.107 & 1.347 & 0.181 & & & \\
\hline Diabetes mellitus (\%) & -0.114 & 1.404 & 0.163 & & & \\
\hline Current smoking (\%) & -0.148 & 1.995 & 0.048 & -0.002 & 0.018 & 0.985 \\
\hline Family history of CAD (\%) & -0.280 & 2.211 & 0.029 & -0.276 & 2.536 & 0.012 \\
\hline $\mathrm{BMI}\left(\mathrm{kg} / \mathrm{m}^{2}\right)$ & -0.015 & 1.570 & 0.119 & & & \\
\hline $\mathrm{TG}(\mathrm{mmol} / \mathrm{l})$ & 0.006 & 0.286 & 0.775 & & & \\
\hline $\mathrm{TC}(\mathrm{mmol} / \mathrm{l})$ & 0.003 & 0.111 & 0.912 & & & \\
\hline HDL-c (mmol/l) & 0.369 & 3.168 & 0.002 & 0.157 & 1.457 & 0.148 \\
\hline LDL-c (mmol/l) & -0.051 & 1.541 & 0.126 & & & \\
\hline$L p(a)(g / L)$ & 0.001 & 1.640 & 0.103 & & & \\
\hline hs-CRP (mg/L) & -0.005 & 2.718 & 0.007 & -0.005 & 2.621 & 0.010 \\
\hline IL-2 (pg/ml) & 0.011 & 0.394 & 0.695 & & & \\
\hline IL-4 (pg/ml) & 0.042 & 1.286 & 0.203 & & & \\
\hline IL-6 (pg/ml) & 0.001 & 0.423 & 0.673 & & & \\
\hline IL-10 (pg/ml) & -0.004 & -0.187 & 0.852 & & & \\
\hline Fibrinogen (g/L) & -0.097 & 3.233 & 0.002 & -0.049 & 1.612 & 0.110 \\
\hline Average heart rate (beats/min) & -0.001 & 0.103 & 0.918 & & & \\
\hline Total power $\left(\mathrm{ms}^{2}\right)$ & 0.001 & 0.070 & 0.944 & & & \\
\hline $\mathrm{LF}\left(\mathrm{ms}^{2}\right)$ & -0.001 & 5.705 & $<0.001$ & -0.001 & 3.128 & 0.002 \\
\hline $\mathrm{HF}\left(\mathrm{ms}^{2}\right)$ & -0.001 & 1.527 & 0.129 & & & \\
\hline LF/HF & -0.09 & 1.137 & 0.258 & & & \\
\hline
\end{tabular}

The meaning of the bold values are a two-sided $P<0.05$ was considered statistically significant.

the discriminatory and reclassification indices for the occurrence of the functional severity of coronary stenosis of target vessel (AUC: 0.904; C-index: 0.913; Youden index: 0.672; sensitivity: 92.2\%; specificity: 75.0\%; CI: 0.852-0.956; $P<0.001$ ) (Figure 6).

\section{DISCUSSION}

For patients with new-onset UAP, we found that (1) plasma hsCRP and LF were independently associated with the functional severity of coronary artery stenosis of target vessel, as well as the total atherosclerotic burden, as determined by cQFR, and LF was positively correlated with hs-CRP; (2) higher plasma hsCRP at baseline was independently correlated with the severity of coronary artery lesion as evaluated by GS, while LF was not associated with GS; and (3) integration of the increase of LF into hs-CRP and GS significantly increased the discriminatory ability and accuracy in the prediction of the functional severity of coronary artery stenosis of target vessel.

\section{HRV and cQFR Measurement}

Although coronary angiogram is the gold standard for diagnosing $\mathrm{CAD}$, the degree of anatomical stenosis of the coronary artery does not always reflect the severity of coronary myocardial ischemia $(3,4,26,27)$. cQFR is a new diagnostic method for the functional evaluation of coronary artery stenosis that can precisely predict the clinical outcome of patients with ACS $(17,26,27)$. Notably, assessment of the physiological functions of 'non-culprit' vessels could help predict future 


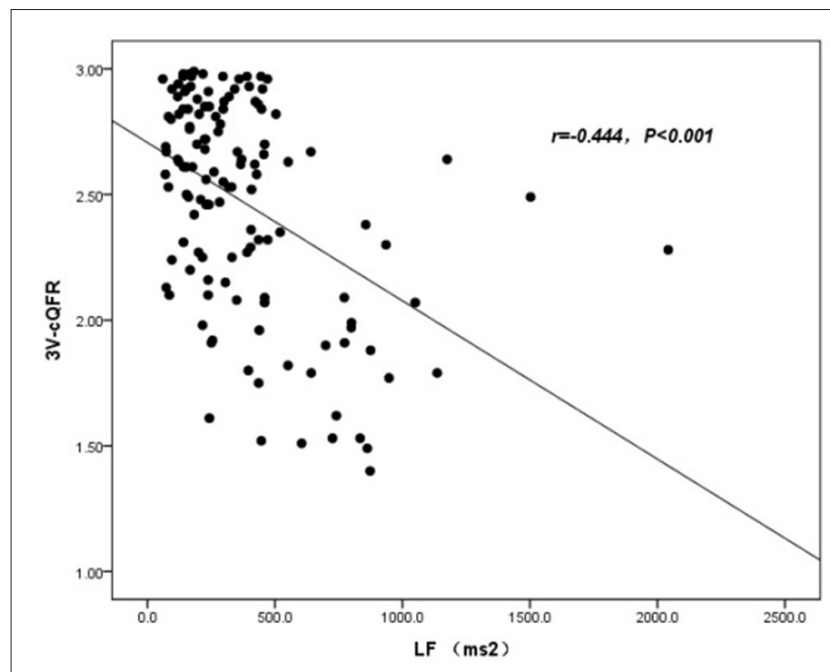

FIGURE 3 | Association analysis between 3V-cQFR and LF.

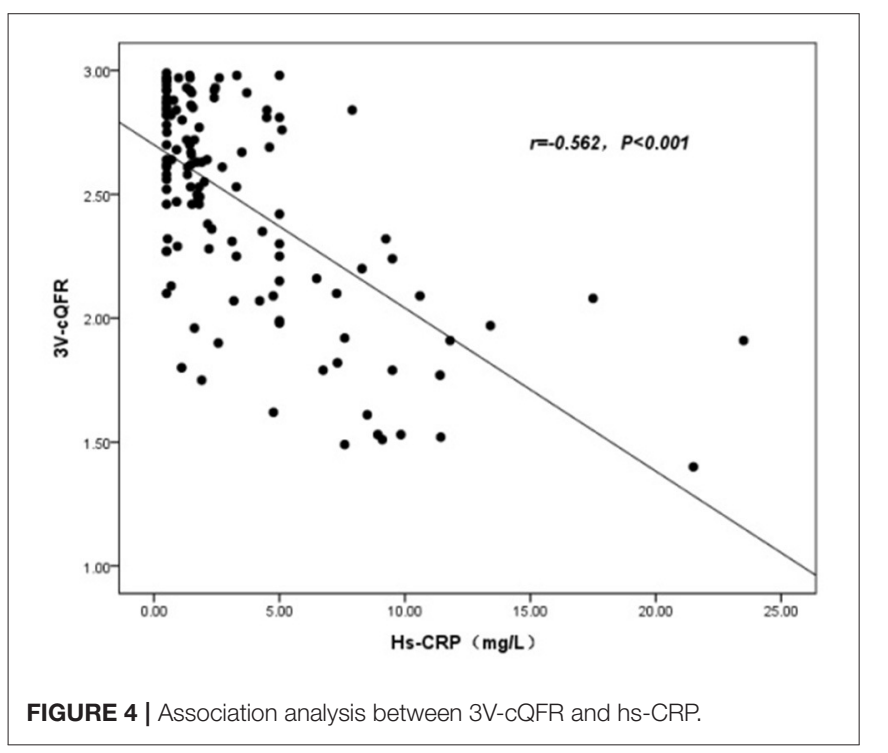

cardiovascular adverse events in "vulnerable" patients even if they are symptom-free $(6,28,29)$. Previous studies have provided new insights into the total "anatomical" atherosclerotic burden (6), and we believe it is reasonable to suggest that $3 \mathrm{~V}-\mathrm{cQFR}$ can be used as an index for total "physiological" atherosclerotic burden and identification of "vulnerable" patients. Moreover, previous studies suggested that cardiac sympathetic nerve modulation reflected by HRV could accelerate coronary atherosclerosis via its pro-inflammatory and prothrombotic effects $(13,30)$. Thus, HRV in patients with no known history of CAD may serve as a biomarker for myocardial ischemia (31). Our study further showed that LF and LFn, reflecting cardiac sympathetic nerve modulation, were associated with functional ischemia, and LF was an independent risk factor for evaluating the functional stenosis of the target vessels and total atherosclerotic burden. Reports have shown that non-cardiovascular pathological conditions, such as respiratory, neurologic, and renal disease, can affect HRV $(23,32)$. In addition, the current approaches applied to analyze the long-term HRV, such as the 24-h heart rate, are based on the stationary assumption (33-37). However, these methods are still widely used for non-stationary time series, including the $24-48$-h long-term heart rate $(23,38)$. In our study, the average 5-minute short term HRV was used to analyze normal sinus rhythm, as this method has been used an indicator of the combined effect of cardiac regulation by baroreflex, neurohormones, physical and mental activities, and circadian rhythm of daily living. Compared with 24-h (long-term) analysis, we used the relatively accurate method to obtain indexes in the frequency-domain parameters $(23,38)$. Moreover, rigorous inclusion and exclusion criteria were adopted in our study to reduce the influence of HRV (23).

Since HRV is closely correlated with CAD and cardiogenic death, and past CAD may affect HRV that ultimately affects the accuracy of our conclusions, patients with a history of CAD were excluded from our study. Moreover, to the best of our knowledge, the correlation between HRV and new-onset UAP has not been established. Previous studies have shown correlations between HRV and the development and progression of atherosclerosis (12), and low HRV has been identified as an independent predictor for cardiovascular mortality and sudden cardiac death $(21,39)$. Our results are inconsistent with previous studies showing that lower LF was associated with the severity of coronary lesion in patients who underwent angiography (12). Of note, coronary arteriography was intended to evaluate the severity of coronary artery lesions but not the functional severity of coronary artery stenosis. Interestingly, using HRV analysis combined with the HeartTrends DyDx algorithm, another study showed that HRV was correlated with myocardial ischemia through exercise stress echocardiography and exercise myocardial perfusion imaging (31). However, all patients who did not undergo coronary angiography assessing the coronary artery stenosis were included in that study. Likewise, patients with myocardial ischemia were shown to have heightened sympathetic modulation relative to parasympathetic modulation, which suggested that augmented sympathetic modulation is the burden of ischemia (40). Moreover, accelerated heart rate is an early marker of myocardial ischemia $(41,42)$. Since QFR yields a consistent evaluation of FFR that can be used to evaluate functional ischemia of target vessel and total atherosclerotic burden using the $3 \mathrm{~V}$-cQFR measurement $(6,17,26)$, our study provides a more accurate association of these factors. Contrary to previous studies that LF was inversely correlated with the severity of coronary stenosis $(12,21,39)$, we found that LF was directly correlated with the functional severity of coronary artery stenosis. Therefore, we speculate that, in the early stages of myocardial ischemia, there is elevated sympathetic nerve tone in patients with new-onset UAP, leading to an increase in LF, a potential early marker of myocardial ischemia. However, autonomic dysfunction may occur as the CAD progresses, which may lead to a concomitant reduction in LF.

\section{Inflammation and cQFR Measurement}

Currently, the "inflammatory hypothesis of atherothrombosis" is the popular theory of atherosclerosis pathogenesis $(43,44)$. 


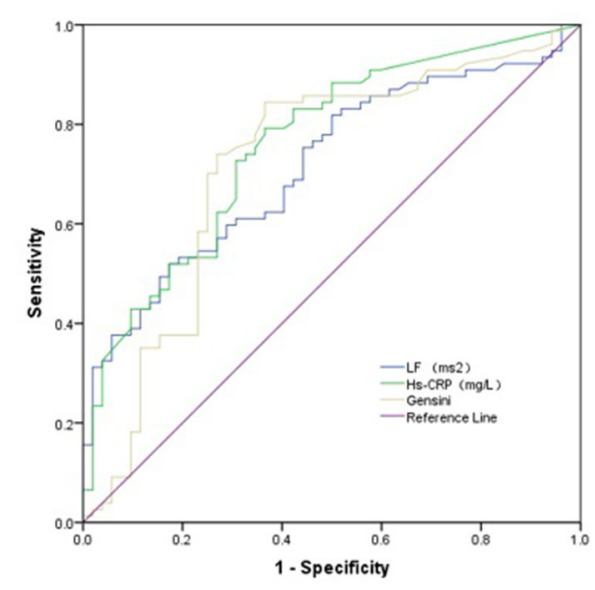

\begin{tabular}{llllllll}
\hline Variables & AUC & SE & P & $95 \%$ CI & Cut-off & Sensitivity & Specificity \\
\hline Gensini & 0.719 & 0.049 & $<0.001$ & $0.623-0.815$ & 17.00 & $84.4 \%$ & $63.5 \%$ \\
Hs-CRP & 0.759 & 0.043 & $<0.001$ & $0.676-0.843$ & 1.45 & $79.2 \%$ & $59.5 \%$ \\
LF & 0.716 & 0.045 & $<0.001$ & $0.628-0.804$ & 390.05 & $51.9 \%$ & $82.7 \%$ \\
\hline
\end{tabular}

FIGURE 5 | ROC analysis for the predictive efficacy of variables for functional ischemia of target vessel as detected by cQFR.

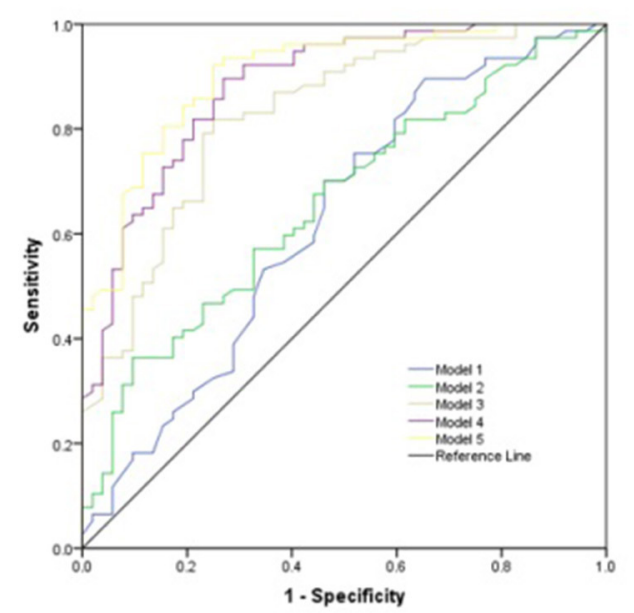

\begin{tabular}{cccccccccc}
\hline Model & AUC & SE & P & OR & $95 \%$ CI & Sensitivity & Specificity & Youden index & C-index \\
\hline Model 1 & 0.625 & 0.051 & 0.016 & 1.851 & $0.525-0.726$ & $89.6 \%$ & $34.6 \%$ & 0.242 & 0.637 \\
Model 2 & 0.658 & 0.048 & 0.002 & 3.055 & $0.564-0-.752$ & $57.1 \%$ & $67.3 \%$ & 0.244 & 0.682 \\
Model 3 & 0.828 & 0.037 & $<0.001$ & 4.957 & $0.756-0.899$ & $81.8 \%$ & $75.0 \%$ & 0.568 & 0.944 \\
Model 4 & 0.891 & 0.030 & $<0.001$ & 7.173 & $0.822-0.940$ & $89.6 \%$ & $73.1 \%$ & 0.627 & 0.897 \\
Model 5 & 0.904 & 0.026 & $<0.001$ & 9.388 & $0.852-0.956$ & $92.2 \%$ & $75.0 \%$ & 0.672 & 0.913 \\
\hline
\end{tabular}

FIGURE 6 | Comparison of discrimination and reclassification abilities of predictive models for functional ischemia of target vessel as detected by cQFR. Model 1 , Age + Sex; Model 2, Model $1+$ Hypertension + Diabetes mellitus + Current smoking + Current drinking + BMl > 28 Kg/m²; Model 3 , Model 2 + Genisi Score > 17; Model 4, Model $3+$ hs-CRP > 1.45 mg/L; Model 5, Model $4+$ LF > $390.5 \mathrm{~ms}^{2}$. 


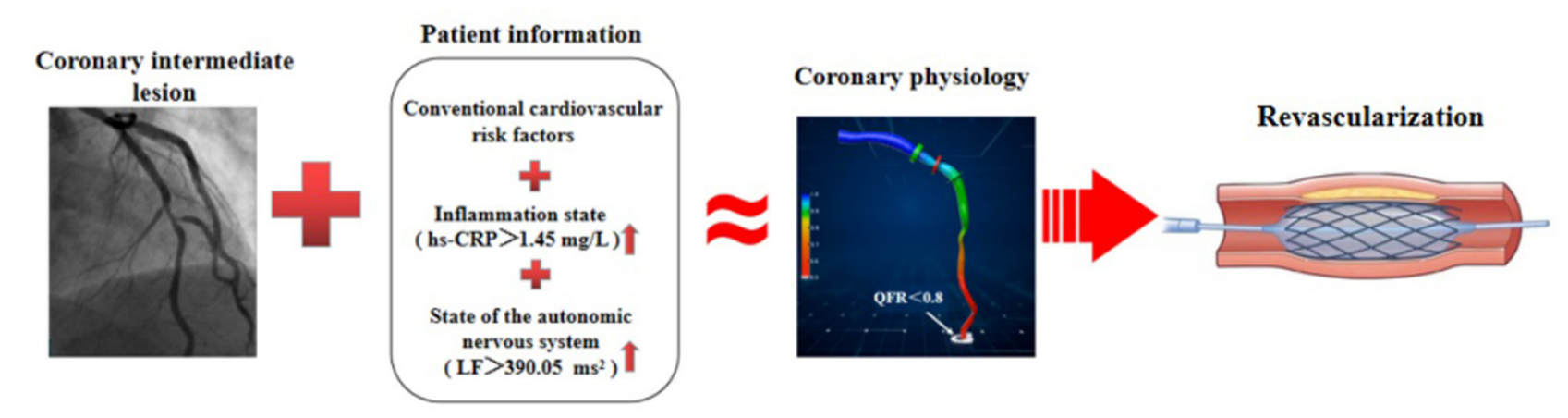

FIGURE 7 | Schematic diagram for evidence to diagnose functional ischemia. In the absence of QFR analysis, model-based risk assessment including LF, hs-CRP, GS, and traditional risk factors, can predict functional ischemia in patients with coronary intermediate lesions and inform whether or not to schedule PCl.

Studies have demonstrated that an increase in hs-CRP and IL6 may confer similar risk as conventional cardiovascular risk factors for the incidence and prognostication of myocardial infarction and atherosclerosis (19, 45-47). Mounting literature acknowledges the importance of evaluating the association between biomarkers of inflammation and CAD risk, which predicts the severity of coronary stenosis and clinical outcomes. In addition, a previous study showed that inflammation might be the triggering mechanism in most, but not all, patients with ACS, suggesting that there are individual differences in the inflammatory response in patients with ACS (48). We used QFR to evaluate not only functional ischemia of target vessel but also the total atherosclerotic burden, including the functional evidence of the non-culprit vessels. We uncovered the pathophysiological basis underlying the association between biomarkers of inflammation and coronary artery events by showing that hs-CRP is associated with the functional severity of coronary artery stenosis. (32). Furthermore, hs-CRP independently predicted the severity of coronary artery lesions by GS, as evidenced by multiple stepwise logistics regression analysis in patients with new-onset UAP. Therefore, our data support that an increased hs-CRP may be an early marker of myocardial ischemia and is directly correlated with the severity of cardiovascular disease.

\section{Autonomic Nervous System and Inflammation}

Emerging evidence demonstrates that the autonomic nervous system modulates inflammatory responses (32). Recently, observational and translational studies found that in response to environmental noise, the sympathetic nervous system becomes activated, which activates several pro-inflammatory pathways, leading to vascular inflammation and endothelial injury that accelerate lipid deposition and recruitment of more inflammatory cells into blood vessels, and thus the development of CAD (14). Our results showed that LF was directly associated with hs-CRP, which is consistent with previous studies showing that multiple mechanisms, especially inflammation, were responsible for the potential role of sympathetic excitation in accelerating coronary artery atherosclerosis (14-16). Importantly, given that model-based risk assessment including LF, hs-CRP, GS, and traditional risk factors can predict functional ischemia in patients with new-onset UAP, non-urgent stent implantations and other dispensable revascularization procedures can be deferred, even in the absence of QFR analysis (Figure 7). These findings indicate that an imbalance in cardiac autonomic control is correlated with increased systemic inflammation in patients with new-onset UAP. Our data also confirmed that excessive sympathetic activation promotes the proliferation, differentiation, and mobilization of bone marrow hematopoietic stem cells and progenitor cells, and increases the number of pro-inflammatory monocytes in circulation, which has been shown to accelerate atherosclerosis (14-16, 49). More studies are required to validate our findings and confirm the importance of monitoring the autonomic nervous system and systemic inflammation in patients with UAP.

\section{Study Limitations}

There are some limitations in our study. First, this was a retrospective observational study with a relatively small sample size from a single center. Thus, we could not prevent selection bias. The findings should be validated in prospective studies with larger samples from multiple centers. Second, the majority of cQFR computation required manual correction for tracing, and the present study did not use FFR as a gold standard for the control group, which could reduce the generalizability of study. Third, since almost one third of selected patients were diabetic and observational data showed discordance between CQFR and FFR in patients with diabetes, the functional ischemia in patients with UAP needs to be further verified using the gold-standard FFR procedure. Fourth, our study did not use optical coherence tomography or intravascular ultrasound to analyze plaque burden or characteristics, and $3 \mathrm{~V}$-cQFR could not represent the total plaque burden because diseased side branches and ostial lesions were not analyzed. Finaly, a lack of longitudinal follow-up data prohibited assessment of the clinical impact of QFR analysis on future events. 


\section{CONCLUSIONS}

In patients with UAP, higher LF and hs-CRP were independently associated with increased risk of functional ischemia and total atherosclerotic burden, as evaluated by cQFR. An imbalance of cardiac autonomic regulation was related to accelerated systemic inflammation in patients with new-onset UAP. Measurement of HRV and hs-CRP may add valuable information for the early diagnosis of functional ischemia and serve as a reliable parameter to decide whether or not to schedule percutaneous transluminal coronary intervention for patients with new-onset UAP, even in the absence of QFR analysis.

\section{DATA AVAILABILITY STATEMENT}

The datasets used and/or analyzed during this study are available from the corresponding author on reasonable request. Requests to access these datasets should be directed to Hong Jiang, hong-jiang@whu.edu.cn.

\section{ETHICS STATEMENT}

Because this was a retrospective observational study, the Renmin Hospital of Wuhan University Ethics Committee granted an

\section{REFERENCES}

1. Khot UN, Khot MB, Bajzer CT, Sapp SK, Ohman EM, Brener SJ, et al. Prevalence of conventional risk factors in patients with coronary heart disease. JAMA. (2003) 290:898-904. doi: 10.1001/jama.290. 7.898

2. Greenland P, Knoll MD, Stamler J, Neaton JD, Dyer AR, Garside DB, et al. Major risk factors as antecedents of fatal and nonfatal coronary heart disease events. JAMA. (2003) 290:891-7. doi: 10.1001/jama.290.7.891

3. Iskandrian AS, Chae SC, Heo J, Stanberry CD, Wasserleben V, Cave $\mathrm{V}$, et al. Independent and incremental prognostic value of exercise single-photon emission computed tomographic (SPECT) thallium imaging in coronary artery disease. J Am Coll Cardiol. (1993) 22:665-70. doi: 10.1016/0735-1097(93)90174-Y

4. Hachamovitch R, Berman DS, Kiat H, Cohen I, Friedman JD, Shaw LJ. Value of stress myocardial perfusion single photon emission computed tomography in patients with normal resting electrocardiograms: an evaluation of incremental prognostic value and cost-effectiveness. Circulation. (2002) 105:823-9. doi: 10.1161/hc0702.103973

5. van de Hoef TP, Siebes M, Spaan JA, Piek JJ. Fundamentals in clinical coronary physiology: why coronary flow is more important than coronary pressure. Eur Heart J. (2015) 36:3312-9a. doi: 10.1093/eurheartj/ehv235

6. Hamaya R, Hoshino M, Kanno Y, Yamaguchi M, Ohya H, Sumino Y, et al. Prognostic implication of three-vessel contrast-flow quantitative flow ratio in patients with stable coronary artery disease. EuroIntervention. (2019) 15:1808. doi: 10.4244/EIJ-D-18-00896

7. Tsuji H, Larson MG, Venditti FJ, Manders ES, Evans JC, Feldman $\mathrm{CL}$, et al. Impact of reduced heart rate variability on risk for cardiac events. The framingham heart study. Circulation. (1996) 94:2850-5. doi: 10.1161/01.CIR.94.11.2850

8. Shah AS, El Ghormli L, Vajravelu ME, Bacha F, Farrell RM, Gidding SS, et al. Heart rate variability and cardiac autonomic dysfunction: prevalence, risk factors, and relationship to arterial stiffness in the treatment options for type 2 diabetes in adolescents and youth (TODAY) study. Diabetes Care. (2019) 42:2143-50. doi: 10.2337/dc19-0993 exemption from requiring ethics approval and informed consent from eligible patients was waived.

\section{AUTHOR CONTRIBUTIONS}

LY and HJ: substantial contributions to conception and design, data acquisition, or data analysis and interpretation. JW, WL, $\mathrm{HuC}, \mathrm{CL}, \mathrm{MW}, \mathrm{HuaC}, \mathrm{HZ}, \mathrm{ZL}, \mathrm{SZ}, \mathrm{ZY}, \mathrm{SD}$, QD, and JS: drafting the article or critically revising it for important intellectual content. JW, WL, HuaC, CL, and MW: final approval of the version to be published and agreement to be accountable for all aspects of the work in ensuring that questions related to the accuracy or integrity of the work are appropriately investigated and resolved. All authors contributed to the article and approved the submitted version.

\section{FUNDING}

This work was supported by the National Key R\&D Program of China [2017YFC1307800], and the National Natural Science Foundation of China [81530011, 81770364, 81871486, and 81700444].

\section{ACKNOWLEDGMENTS}

We thank to everyone for supporting our final study.

9. Kataoka M, Ito C, Sasaki H, Yamane K, Kohno N. Low heart rate variability is a risk factor for sudden cardiac death in type 2 diabetes. Diabetes Res Clin Pract. (2004) 64:51-8. doi: 10.1016/j.diabres.2003.10.009

10. van Boven AJ, Jukema JW, Haaksma J, Zwinderman AH, Crijns HJ, Lie KI. Depressed heart rate variability is associated with events in patients with stable coronary artery disease and preserved left ventricular function. REGRESS Study Group. Am Heart J. (1998) 135:571-6. doi: 10.1016/S0002-8703(98)70269-8

11. Singh JP, Larson MG, Tsuji H, Evans JC, O’Donnell CJ, Levy D. Reduced heart rate variability and new-onset hypertension: insights into pathogenesis of hypertension: the Framingham Heart Study. Hypertension. (1998) 32:2937. doi: 10.1161/01.HYP.32.2.293

12. Kotecha D, New G, Flather MD, Eccleston D, Pepper J, Krum H. Five-minute heart rate variability can predict obstructive angiographic coronary disease. Heart. (2012) 98:395-401. doi: 10.1136/heartjnl-2011-300033

13. von Känel R, Carney RM, Zhao S, Whooley MA. Heart rate variability and biomarkers of systemic inflammation in patients with stable coronary heart disease: findings from the Heart and Soul Study. Clin Res Cardiol. (2011) 100:241-7. doi: 10.1007/s00392-010-0236-5

14. Münzel T, Schmidt FP, Steven S, Herzog J, Daiber A, Sørensen M. Environmental noise and the cardiovascular system. J Am Coll Cardiol. (2018) 71:688-97. doi: 10.1016/j.jacc.2017.12.015

15. Dutta P, Courties G, Wei Y, Leuschner F, Gorbatov R, Robbins CS. Myocardial infarction accelerates atherosclerosis. Nature. (2012) 487:3259. doi: 10.1038/nature11260

16. Vaccarino V, Badimon L, Bremner JD, Cenko E, Cubedo J, Dorobantu M, et al. Depression and coronary heart disease: 2018 position paper of the ESC working group on coronary pathophysiology and microcirculation. Eur Heart J. (2020) 41:1687-96. doi: 10.1093/eurheartj/ehy913

17. Xu B, Tu S, Qiao S, Qu X, Chen Y, Yang J, et al. Diagnostic accuracy of angiography-based quantitative flow ratio measurements for online assessment of coronary stenosis. J Am Coll Cardiol. (2017) 70:307787. doi: 10.1016/j.jacc.2017.10.035

18. Kogame N, Ono M, Kawashima H, Tomaniak M, Hara H, Leipsic J, et al. The impact of coronary physiology on contemporary clinical decision 
making. JACC Cardiovasc Interv. (2020) 13:1617-38. doi: 10.1016/j.jcin.2020. 04.040

19. Tajfard M, Tavakoly Sany SB, Avan A, Latiff LA, Rahimi HR, Moohebati M, et al. Relationship between serum high sensitivity C-reactive protein with angiographic severity of coronary artery disease and traditional cardiovascular risk factors. J Cell Physiol. (2019) 234:10289-99. doi: 10.1002/jcp.27945

20. Mendis S, Thygesen K, Kuulasmaa K, Giampaoli S, Mähönen M, Ngu Blackett $\mathrm{K}$, et a1. Wodd Health Organization definition of myocardial infarction:200809 revision. Int J Epidemiol. (2011) 40:139-46. doi: 10.1093/ije/dyq165

21. Voss A, Schroeder R, Vallverdú M, Schulz S, Cygankiewicz I, Vázquez R, et al. Short-term vs. long-term heart rate variability in ischemic cardiomyopathy risk stratification. Front Physiol. (2013) 4:364. doi: 10.3389/fphys.2013.00364

22. Catai AM, Pastre CM, Godoy MF, Silva ED, Takahashi ACM, Vanderlei LCM. Heart rate variability: are you using it properly? Standardisation checklist of procedures. Braz J Phys Ther. (2020) 24:91-102. doi: 10.1016/j.bjpt.2019.02.006

23. Taralov ZZ, Terziyski KV, Kostianev SS.. Heart rate variability asa method for assessment of the autonomic nervous system and the adaptations to different physiological and pathological conditions. Folia Med. (2015) 57:17380. doi: 10.1515/folmed-2015-0036

24. Xhyheri B, Manfrini O, Mazzolini M, Pizzi C, Bugiardini R. Heart rate variability today. Prog Cardiovasc Dis. (2012) 55:321-31. doi: 10.1016/j.pcad.2012.09.001

25. Gensini GG. A more meaningful scoring system for determining the severity of coronary heart disease. Am J Cardiol. (1983) 51:606. doi: 10.1016/S0002-9149(83)80105-2

26. Westra J, Tu S, Winther S, Nissen L, Vestergaard MB, Andersen BK, et al. Evaluation of coronary artery stenosis by quantitative flow ratio during invasive coronary angiography: the WIFI II study (Wire-Free Functional Imaging II). Circ Cardiovasc Imaging. (2018) 11:e007107. doi: 10.1161/CIRCIMAGING.117.007107

27. Foy AJ, Dhruva SS, Peterson B, Mandrola JM, Morgan DJ, Redberg RF. Coronary computed tomography angiography vs functional stress testing for patients with suspected coronary artery disease: a systematic review and meta-analysis. JAMA Intern Med. (2017) 177:1623-31. doi: 10.1001/jamainternmed.2017.4772

28. Shan P, Mintz GS, McPherson JA, De Bruyne B, Farhat NZ, Marso SP et al. Usefulness of coronary atheroma burden to predict cardiovascular events in patients presenting with acute coronary syndromes (from the PROSPECT study). Am J Cardiol. (2015) 116:1672-7. doi: 10.1016/j.amjcard.2015.08.038

29. Lee JM, Koo BK, Shin ES, Nam CW, Doh JH, Hwang D, et al. Clinical implications of three-vessel fractional flow reserve measurement in patients with coronary artery disease. Eur Heart J. (2018) 39:94551. doi: 10.1093/eurheartj/ehx458

30. Madsen T, Christensen JH, Toft E, Schmidt EB. C-reactive protein is associated with heart rate variability. Ann Noninvasive Electrocardiol. (2007) 12:216-22. doi: 10.1111/j.1542-474X.2007.00164.x

31. Goldenberg I, Goldkorn R, Shlomo N, Einhorn M, Levitan J, Kuperstein R et al. Heart rate variability for risk assessment of myocardial ischemia in patients without known coronary artery disease: the HRV-DETECT (Heart Rate Variability for the Detection of Myocardial Ischemia) study. J Am Heart Assoc. (2019) 8:e014540. doi: 10.1161/JAHA.119.014540

32. Williams DP, Koenig J, Carnevali L, Sgoifo A, Jarczok MN, Sternberg EM et al. Heart rate variability and inflammation: a meta-analysis of human studies. Brain Behav Immun. (2019) 80:219-26. doi: 10.1016/j.bbi.2019.03.009

33. Shahbazi F, Asl BM. Generalized discriminant analysis for congestive heart failure risk assessment based on longterm heart rate variability. Comput Methods Progr Biomed. (2015) 122:191-8. doi: 10.1016/j.cmpb.2015.08.007

34. Melillo P, De Luca N, Bracale M, Pecchia L. Classifcation tree for risk assessment in patients sufering from congestive heart failure via longterm heart rate variability. IEEE J Biomed Health Inform. (2013) 17:72733. doi: 10.1109/JBHI.2013.2244902

35. Flachenecker P, Lem K, Mullges W, Reiners K. Detection of serious bradyarrhythmias in Guillain-Barre syndrome: sensitivity and specifcity of the 24-h heart rate power spectrum. Clin Auton Res. (2000) 10:18591. doi: 10.1007/BF02291354

36. Melillo P, Fusco R, Sansone M, Bracale M, Pecchia L. Discrimination power of long-term heart rate variability measures for chronic heart failure detection. Med Biol Eng Comput. (2011) 49:67-74. doi: 10.1007/s11517-010-0728-5
37. Fei L, Copie X, Malik M, Camm AJ. Short- and long-term assessment of heart rate variability for risk stratifcation after acute myocardial infarction. Am J Cardiol. (1996) 1996:681-4. doi: 10.1016/S0002-9149(97)89199-0

38. Wu HT, Soliman EZ. A new approach for analysis of heart rate variability and QT variability in long-term ECG recording. Biomed Eng Online. (2018) 17:54. doi: 10.1186/s12938-018-0490-8

39. Dekker JM, Crow RS, Folsom AR, Hannan PJ, Liao D, Swenne CA, et al. Low heart rate variability in a 2-minute rhythm strip predicts risk of coronary heart disease and mortality from several causes: the ARIC Study. Atherosclerosis Risk In Communities. Circulation. (2000) 102:123944. doi: 10.1161/01.CIR.102.11.1239

40. Sharma R, O'Driscoll JM, Saha A, Sritharan M, Sutton R, Rosen SD. Differing autonomic responses to dobutamine stress in the presence and absence of myocardial ischaemia. J Physiol. (2015) 593:2171-84. doi: 10.1113/JP270063

41. Lang CC, Gupta S, Kalra P, Keavney B, Menown I, Morley C, et al. Elevated heart rate and cardiovascular outcomes in patients with coronary artery disease: clinical evidence and pathophysiological mechanisms. Atherosclerosis. (2010) 212:1-8. doi: 10.1016/j.atherosclerosis.2010.01.029

42. Perna GP, Battistoni I, Angelini L. Heart rate modulation in stable ischemic heart disease: what we have learned from the SIGNIFY study? G Ital Cardiol. (2015) 16:155-60. doi: 10.1714/1820.19824

43. Ridker PM, Everett BM, Thuren T, MacFadyen JG, Chang WH, Ballantyne C, et al. Antiinflammatory therapy with canakinumab for atherosclerotic disease. N Engl J Med. (2017) 377:1119-31. doi: 10.1056/NEJMoa1707914

44. Goldfine Allison B,Shoelson Steven E.Therapeutic approaches targeting inflammation for diabetes and associated cardiovascular risk. J Clin Invest. (2017) 127:83-93. doi: 10.1172/JCI88884

45. Shitara J, Ogita M, Wada H, Tsuboi S, Endo H, Doi S, et al. Clinical impact of high-sensitivity C-reactive protein during follow-up on longterm adverse clinical outcomes in patients with coronary artery disease treated with percutaneous coronary intervention. J Cardiol. (2019) 73:4550. doi: $10.1016 /$ j.jjcc.2018.06.002

46. Walter J, Tanglay Y, du Fay de Lavallaz J, Strebel I, Boeddinghaus J, Twerenbold R, et al. Clinical utility of circulating interleukin- 6 concentrations in the detection of functionally relevant coronary artery disease. Int J Cardiol. (2019) 275:20-5. doi: 10.1016/j.ijcard.2018.10.029

47. Groot HE, Al Ali L, van der Horst ICC, Schurer RAJ, van der Werf HW, Lipsic E, et al. Plasma interleukin 6 levels are associated with cardiac function after ST-elevation myocardial infarction. Clin Res Cardiol. (2019) 108:61221. doi: $10.1007 / \mathrm{s} 00392-018-1387-\mathrm{z}$

48. Cristell N, Cianflone D, Durante A, Ammirati E, Vanuzzo D, Banfi M, et al. High-sensitivity C-reactive protein is within normal levels at the very onset of first ST-segment elevation acute myocardial infarction in $41 \%$ of cases: a multiethnic case-control study. J Am Coll Cardiol. (2011) 58:265461. doi: 10.1016/j.jacc.2011.08.055

49. Yu L, Huang B, Po SS, Tan T, Wang M, Zhou L, et al. Low-level tragus stimulation for the treatment of ischemia and reperfusion injury in patients with ST-segment elevation myocardial infarction: a proof-of-concept study. JACC-Cardiovasc Interv. (2017) 10:1511-20. doi: 10.1016/j.jcin.2017. 04.036

Conflict of Interest: The authors declare that the research was conducted in the absence of any commercial or financial relationships that could be construed as a potential conflict of interest.

Publisher's Note: All claims expressed in this article are solely those of the authors and do not necessarily represent those of their affiliated organizations, or those of the publisher, the editors and the reviewers. Any product that may be evaluated in this article, or claim that may be made by its manufacturer, is not guaranteed or endorsed by the publisher.

Copyright (๑) 2021 Wang, Liu, Chen, Liu, Wang, Chen, Zhou, Liu, Zhang, Yu, Duan, Deng, Sun, Jiang and Yu. This is an open-access article distributed under the terms of the Creative Commons Attribution License (CC BY). The use, distribution or reproduction in other forums is permitted, provided the original author(s) and the copyright owner(s) are credited and that the original publication in this journal is cited, in accordance with accepted academic practice. No use, distribution or reproduction is permitted which does not comply with these terms. 